\title{
Modified-release Calcifediol Capsule
}

National Cancer Institute

\section{Source}

National Cancer Institute. Modified-release Calcifediol Capsule. NCI Thesaurus. Code

C118789.

An orally available, modified-release formulation containing the calcitriol prohormone, calcifediol (25-hydroxyvitamin D), which can potentially be used for vitamin $D$ supplementation. Upon oral administration of the modified-release calcifediol capsule, calcifediol is slowly and gradually released in the gastrointestinal tract. Then it is taken up by the body and converted, in the kidneys, to the active form calcitriol (1,25dihydroxyvitamin $\mathrm{D}$ or 1,25 D). This form increases and normalizes vitamin $\mathrm{D}$ plasma levels, which, in turn, regulates calcium plasma levels, and normalizes elevated parathyroid hormone (PTH) levels by suppressing both PTH synthesis, and secretion. This formulation appears to have fewer side effects than supplementation with formulations containing active 1,25 D and does not stimulate the upregulation of vitamin D 24-hydroxylase (CYP24), a cytochrome P-450 family enzyme that inactivates vitamin D. 